\title{
Motion Capture Camera System for Measurement of Head and Shoulders Position
}

\author{
J. Hejda ${ }^{1}$, P. Kutilek ${ }^{2}$, J. Hozman ${ }^{1}$, R. Cerny ${ }^{3}$ \\ ${ }^{1}$ Department of Biomedical Technology, Czech Technical University in Prague, Kladno, Czech Republic, \\ jan.hejda@fbmi.cvut.cz \\ ${ }^{2}$ Department of Natural Sciences, Czech Technical University in Prague, Kladno, Czech Republic \\ ${ }^{3}$ Department of Neurology, Charles University in Prague,
}

\section{Structure: $\quad$ 1. Introduction / 2. Methods / 3. Conclusion}

\begin{abstract}
The objective of our study was to develop a technique for precise head and shoulder posture measurement or, in other words, for measuring the native position of the head and shoulders in 3D space with accuracy to $1^{\circ}$. No technique or cheap MoCap camera system has been previously developed that can be widely and easily used in clinical practice.

New technique and MoCap system could have important applications, as there are many neurological disorders that affect the postural alignment position of the head and shoulders. In many cases, the abnormalities of the head position can be small and difficult to observe. Although an accurate method could contribute to the diagnosis of vestibular disorders and some other disorders, this issue was not systematically studied.

MoCap system of head and shoulder posture measurement is based on combination of infrared camera image recognition and evaluation of data recorded by gyroscope. This method was chosen to offer precision less than $1^{\circ}$ in each angle. The system consists of separate components - a headset equipped by gyro-accelerometer and infrared LEDs, infrared LEDs placed on patient's shoulders, and a stand with two infrared cameras. The first camera is placed above the patient and the other in front of him.

Our designed system provides direct information for physicians on the current position of the patient's head and patient's shoulders represented by the angles. Our method is cheaper and offers better application in the field of quantitative assessment of head and shoulder posture.
\end{abstract}

\section{Introduction}

\subsection{The importance of accurate head and shoulders position measuring}

Abnormal head posture (AHP) is an important clinical sign of disease in many medical specialties [1]. There is large number of neurological disorders that affect the postural alignment position of the head and shoulders. These can be divided into three main groups:

- Cervical blockages and diseases of the cervical spine often cause a wide range of positional abnormalities.

- Dystonic "movement disorders". Abnormal body segment position is typical for dystonia.

- Paralyses of eye muscles also often cause a position that attempts to compensate for the insufficient function.

The simultaneous measurement of head and shoulders position could contribute to better definition of diseases affecting the vestibular system (labyrinthine) function in man, [2], [3]. This also allows more precise diagnosis of diseases mentioned above.

Among other it can be used for physical medicine and rehabilitation, especially in the management and diagnosis of disorders affecting cervical spine. Habitual head anteflexion with chronic overload of cervical and upper thoracic spine and muscle imbalance is typical consequence of uncompensated sedentary way of life, starting already in school age.

\subsection{Measurement systems and methods}

Currently, an orthopedic goniometer is used as standard way to simply and rapidly measure angles in clinical practice. However, there are some limitations, especially in the case of head and shoulder posture measurement. Young [4] designed a new method to study head position by mirrors. The main principle of new approach is based on using three mirrors and special head markers. Ferrario, V.F. et al, 1995 [5] developed a new method based on television technology that was faster than conventional analysis. Galardi, G. et al, 2003 [6] developed an objective method for measuring posture using expensive Fastrack system. Meers et al, 2008, [7] developed accurate methods for pinpointing the position of infrared LEDs using an inexpensive USB camera and low-cost algorithms for estimating the 3D coordinates of the LEDs based on known geometry. Charfreitag et al, [8], [9], deal with systems designed for parallel measurement of eye and head positions and a new portable system for studying eye and head movements at the same time. But the systems and methods have the similar disadvantages such as complex preparation, very large sensors or the inability to accurately define the anatomical coordinate system. 
Hozman, J. et al, 2004 [1], [10] proposed a new method based on the application of three digital cameras with stands and appropriate image processing software. The technique aimed to determine differences between the anatomical coordinate system and the physical coordinate system with accuracy from one to two degrees for tilt and rotation. Pictures of the head marked on the tragus and the outer eye canthus are taken simultaneously by three digital cameras aligned by a laser beam, [11]. In our recent method [12], [13], two cameras are required for determining head positions.

The goal of our study is simplification and complete optimization of a technical equipment designed (by Hozman, J. et al, 2004 [1], [13]) and used for a vestibular apparatus examination in otoneurology. More concretely it is a design of a device for head and shoulders position measurement. Because of the insufficient accuracy of the current equipment (only two degrees), the new hardware and software is developed.

Proposed system should be able to determine tilt, rotation and inclination angles in physical coordinate system defined by the vector of the gravitational acceleration. Accuracy of the measurement should be less than one degree in each direction.

\subsection{Reference planes}

Main reference for sagittal plane is called Frankfort horizontal (line connecting meatus acusticus with the orbital floor or line connecting tragus with the outer eye canthus), see Image 2. In most subjects this line is inclined forward bellow the space horizontal, in the extensor type of cervical positions is reclined backwards, [13]. The position of the two anatomical points (acromions) on the shoulders defines the shoulder axis, [14].

The technique of measurement [12] is aimed at determining differences between the anatomical coordinate system and the physical coordinate system with accuracy from one to two degrees for inclination, rotation and angles between the head axis and shoulder axis, [14].
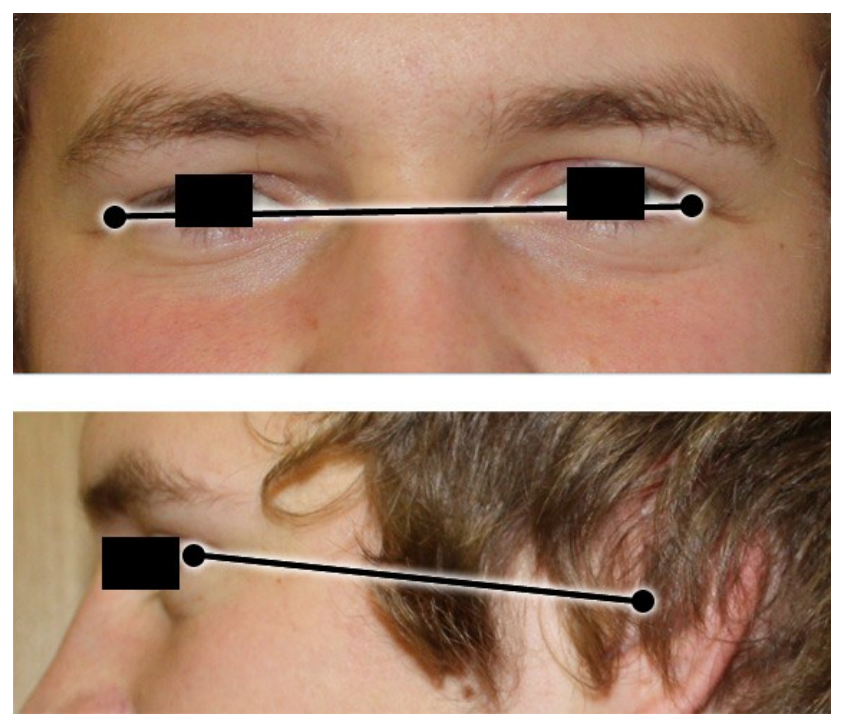

Image 2 Anatomical head axis.

\section{Methods}

Our designed system is composed of a non-invasive examination device and specially developed software. The method of measurement is based on combination of image recognition technique and capturing data from highly accurate gyroscope.

This approach should avoid the biggest disadvantage of gyroscopes - low accuracy of rotation measurement. This is achieved by combination of rotation angle proposed by gyroscope with values determined by image recognition algorithm.

\subsection{Device}

The device is divided into two main components - camera stand and helmet.

Because it is necessary to capture an image from the top and front of the patient, the universal camera stand was made. It holds the cameras on mentioned position and allows its adjustment, Image 1.

The industrial monochromatic cameras Basler has been chosen for image capturing. They offer standard VGA (640x480px) resolution with frame rate 100fps. Because they are used for infrared (IR) LEDs capture, they are supplied by infrared light permeable optical filter Kodak Wratten 2 No. 87 to suppress visible optical spectrum. The cameras are connected to Gigabit PoE (Power over Ethernet) switch which also provides their power supply.

To ensure camera calibration with Earth (i.e. physical) coordinate system, the accelerometers Phidget are mounted

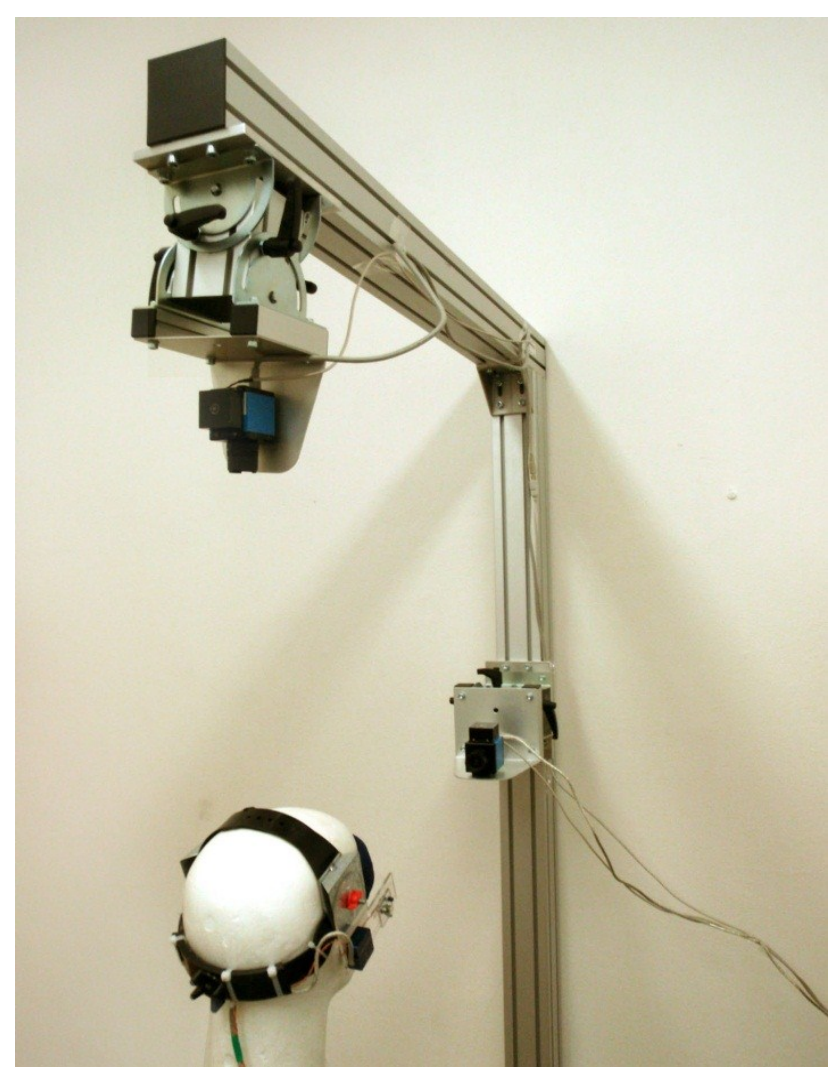

Image 1 Experimental setup for verification of measurement. 


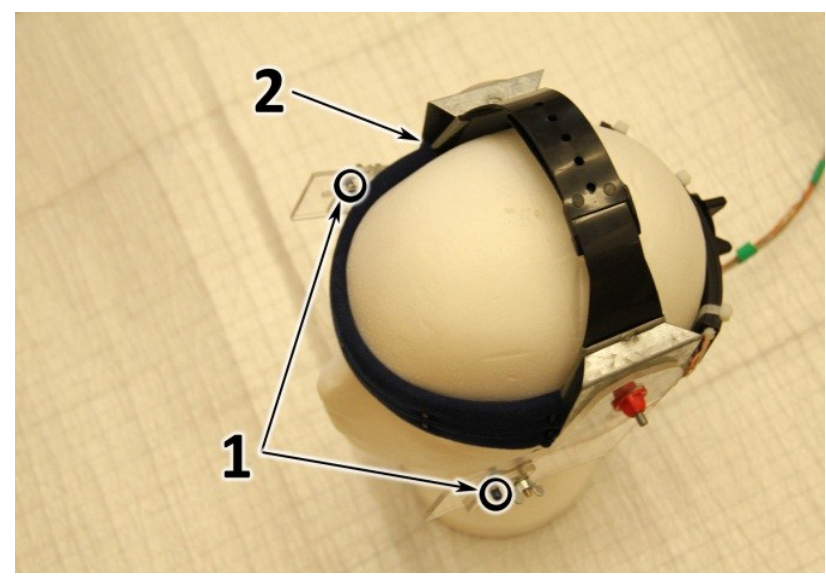

Image 4 Helmet with infrared LEDs (1) and motion tracker (2).

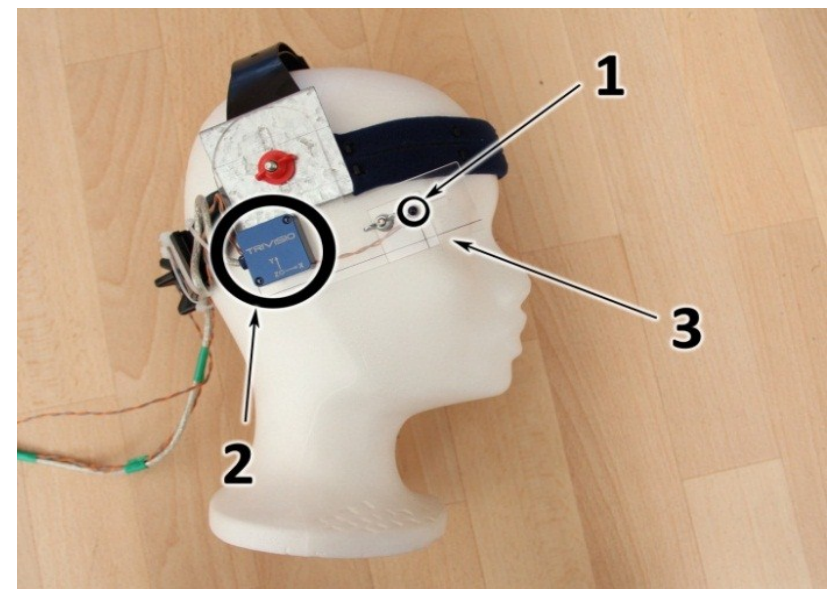

Image 5 Helmet with calibration slider (3), infrared LEDs (1) and motion tracker (2).

to cameras. They are directly connected to operating computer via USB interface.

The helmet is the one of two system components that touches the patient. It contains two infrared LEDs and an inertial motion tracker Trivisio Colibri connected via USB interface, Image 4 and Image 5. This motion tracker contains integrated gyroscope, accelerometer and magnetometer and it is able to provide its tilt and inclination relative to the Earth's reference axis.

Because it is necessary to ensure that the gyroscope is parallel to the Frankfort horizontal, the helmet is equipped with calibration sliders. The role of physician is to set the sliders according to Frankfort horizontal (see above).

The IR diodes placed before examination on patient's shoulders are the second part of system that touches the patient. Position of the two anatomical points (acromions) on the shoulders is defined by these two IR diodes.

\subsection{Software}

Software is designed to offer the highest possible customization. In basic configuration it captures image by two infrared cameras, collects data from accelerometers attached to cameras and reads data from inertial motion tracker.

The standard PC is used as a service computer. It must fulfill the following requirements:
- Gigabit Ethernet interface,

- At least 3 USB ports,

- It must be powerful enough to process video from two cameras in real time.

Due to the limited devices driver support, the Microsoft Windows operating system was chosen. The utility software is built on Microsoft .NET 4.0 platform and implemented in $C \#$ programming language.

As expected with the use of application in clinical practice, it is essential the program was easy to use. For this reason the program is widely configurable and thus autonomous. Further the development was focused on high software modularity to allow flexible support for new sensors and use.

The application is divided into two principal components kernel and GUI. The kernel is represented by application class library offering interface for second component GUI which provides interaction with user.

Proposed software is very variable and it can be preconfigured by $X M L$ to make the simplest possible installation and maintenance.

Thanks to modular design, the application can be extended to support new hardware devices and data processing algorithms.

As mentioned, kernel is an application class library handling the communication with hardware devices (sensors). It grabs and processes the measured data.

Kernel's activity can be divided into the three following phases (dataflow diagram illustrating this process is shown in Image 5):

- Data source - data are loaded from sensor.

- Data filter - specified transformation is applied on data. Application of data filters can be chained or may even be used.

- Data provider - processed data are provided to the GUI.

Each mentioned phase for every specified sensor/filter is performed by corresponding module. Because of the fact

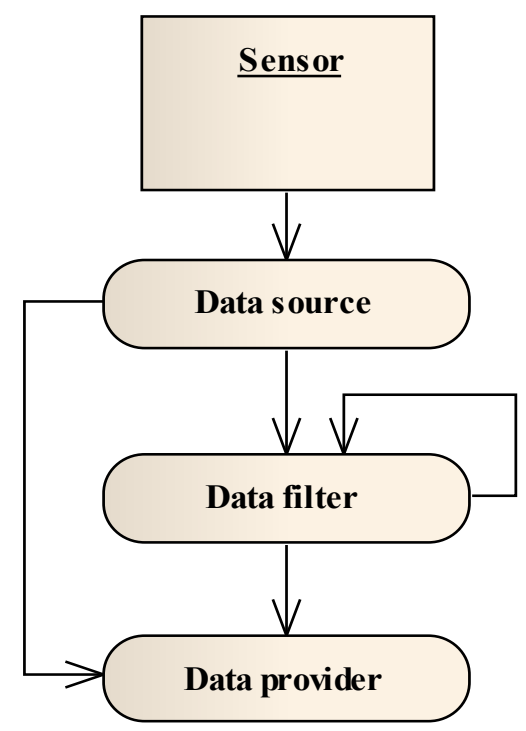

Image 3 Kernel dataflow diagram. 
the data source modules ensures management of sensors and they only provide data to successor data filters and data providers it won't be described in higher detail.

The image recognition is carried out using the data filter module called image to angles module. It gets raw data from camera data module and gives determined angles into the output.

Principle of angles determination is based on marker coordinates (infrared LEDs) in video shots captured by two cameras and a calculation of their mutual position using the formula

$$
\alpha=\tan ^{-1} \frac{y_{2}-y_{1}}{x_{2}-x_{1}}
$$

where $\left[x_{i}, y_{i}\right]$ is a position of marker $i$ in Cartesian coordinate system.

The marker identification in pictures is based on thresholding, distance transform [15] and pattern recognition technique which finds the marker's center.

Because it is necessary to calibrate the system and normalize the measured angles, there is included an angle calibration filter module.

The last module in application dataflow is an angle combiner provider module. It allows assigning the priorities and maximum deviations (relative to other values) to each measured angle. These combined values are subsequently provided to GUI which presents the angles to physician.

\section{Conclusion}

We have designed special equipment and implemented procedures for determining head positions. The new methods have been described in this paper. New equipment and measurement method are designed for a very accurate evaluation of head position in neurological practice. The system is cheaper than sophisticated systems using accelerometers and magnetometers or expensive commercial camera system such as Vicon or Optitrack.

\section{References}

[1] R. Cerny, K. Strohm, J. Hozman, J. Stoklasa and D. Sturm, "Head in Space - Noninvasive Measurement of Head Posture," Bled, 2006.

[2] K. Murphy, C. Preston and W. Evans, "The Development of Instrumentation for the Dynamic Measurement of Changing Head Posture," American Journal of Orthodontics and Dentofacial Orthopedics, vol. 99, no. 6, pp. 520-526, 1991.

[3] S. Raine and L. Twomey, "Head and Shoulder Posture Variations in 160 Asymptomatic Women and Men," Archives of Physical Medicine and Rehabilitation, vol. 78, no. Nov, pp. 1215-1223, 1997.

[4] J. D. Young, "Head Posture Measurement," Journal of Pediatric Ophthalmology and Strabismus, vol. 25, no. Mar-Apr (2), pp. 86-89, 1988.

[5] V. Ferrario, C. Sforza, D. Germann, L. Dalloca and
A. Miani, "Head Posture and Cephalometric Analyses: An Integrated Photographic / Radiographic Technique," American Journal of Orthodontics \& Dentofacial Orthopedics, vol. 106, pp. 257-264, 1994.

[6] G. Galardi, S. Micera, J. Carpaneto, S. Scolari, M. Gambini and P. Dario, "Automated assessment of cervical dystonia," Movement Disorders, vol. 18, no. 11, p. 1358-1367, November 2003.

[7] S. Meers, K. Ward and I. Piper, "Robust and Accurate Head-Pose Tracking Using a Single Camera, Mechatronics and Machine Vision in Practice," Berlin: Springer Science + Business Media, pp. 111-122, 2006.

[8] J. Charfreitag, J. Hozman and R. Cerny, "Measurement of eye and head position in neurological practice," in World Congress on Medical Physics and Biomedical Engineering, Munich, 2009.

[9] J. Charfreitag, J. Hozman and R. Černý, "Specialized glasses - projection displays for neurology investigation," in IFMBE Proceedings, 2008.

[10] J. Hozman, D. Sturm, J. Stoklasa and R. Cerny, "Measurement of Postural Head Alignment in Neurological Practice," in The 3rd European Medical and Biological Engineering Conference - EMBEC'05, Prague, 2005.

[11] J. Hozman, V. Zanchi, R. Cerny, P. Marsalek and Z. Szabo, "Precise Advanced Head Posture Measurement," in The 3rd WSEAS International Conference on Remote Sensing (REMOTE'07), 2007.

[12] P. Kutilek and J. Hozman, "Non-contact method for measurement of head posture by two cameras and calibration means," in The 8th Czech-Slovak Conference on Trends in Biomedical Engineering, Bratislava, 2009.

[13] P. Kutílek, J. Hozman, R. Černý and J. Hejda, "Methods of Measurement and Evaluation of Eye, Head and Shoulders Position in Neurological Practice," in Advanced Topics in Neurological Disorders, Rijeka, InTech; Open Access Company (InTech Europe), 2012, pp. 25-44.

[14] A. Harrison and G. Wojtowicz, "Clinical Measurement of Head and Shoulder Posture Variables," The Journal of Orthopaedic and Sports Physical Therapy (JOSPT), vol. 23, pp. 353-361, 1996.

[15] H. Breu, J. Gil, D. Kirkpatrick and M. Werman, "Linear time Euclidean distance transform algorithms," IEEE Transactions on Pattern Analysis and Machine Intelligence, pp. 529-533, May 1995.

[16] V. Ferrario, C. Sforza, G. Tartaglia, E. Barbini and M. G., "New Television Technique for Natural Head and Body Posture Analysis," Cranio, vol. 13, pp. 247-255, 1995. 